\title{
Comparison and Translation Between English and Chinese from the Perspective of Reception Aesthetics and Aesthetic Linguistics-a Case Study of Character Reports
}

\author{
Liu Yali ${ }^{1, a}$, Dong Mei ${ }^{2, b}$ \\ ${ }^{1,2}$ School of Foreign languages, Xi'an Shiyou University \\ Xi'an, Shanxi Province, 710065, China
}

\begin{abstract}
Character reports, as an important type of news, can convey events and attract readers. News reports, as the channel of information transmission, has distinct language features, and character reports also have unique language features. Chinese and English character news reports are different in syntactic structure, vocabulary selection and many other aspects. Reception aesthetics emphasizes the importance of the reader and takes the reader's acceptance of character translation into consideration. This paper mainly takes the Chinese and English character news reports as examples, making a comparative analysis of the characteristics of Chinese and English character news reports from the perspective of reception aesthetics and aesthetic linguistics, which plays an important guiding role in understanding the differences between English and Chinese, and thus proposes the appropriate translation strategies or skills for such reports to make it easier for target readers' to accept.
\end{abstract}

Keywords-comparison; translation; reception aesthetics; aesthetic linguistics; character reports

\section{INTRODUCTION}

It is a common phenomenon that people make contrasts on things, and languages are no exception. People are often consciously and unconsciously make a contrast among languages. The contrastive method is one of the basic methods in language research. Contrastive linguistics, in a narrow sense, refers to the contrastive study of two or more languages in terms of phonetics, morphemes, words, phrases, sentences, and discourse. In a broad sense, contrastive linguistics also refers to the contrastive research on national thinking, national culture, national history and national psychology, which are closely related to language [1]. Contrastive linguistics is a practice-oriented, structuralism-based linguistic approach that seeks to systematically identify the points of structural similarities and differences between two or more languages at such levels as phonology, grammar and semantics, in order to help solve practical problems in e.g. making foreign language teaching more effective by avoiding negative transfers and having more forethought in the curriculum development, assisting interlingual transfer in translating texts from one language into another, finding lexical equivalents in compiling bilingual dictionaries and nurturing cross-cultural competence increasingly demanded by internationalization.

\section{LITERATURE REVIEW OF COMPARISON AND} TRANSLATION BETWEEN ENGLISH AND CHINESE

\section{A. Overseas Research}

Contrastive linguistics is a branch of modern linguistics, which originated from Europe and America. In 1926, Massehughes published an article on contrastive analysis between English and Czech. Wolfe, an American contrastive linguist, first proposed the concept contrastive linguistics, aiming to study the major differences between different languages in grammatical logic and general empirical analysis. The real founder of contrastive linguistics is Humboldt. In 1820, Humboldt published an article entitled The relationship between linguistic comparative study and different stages of language development, expounding a series of basic theoretical issues of linguistic comparative study. He believed that language was an external embodiment of national spirit and that comparative linguistic study was an accurate exploration of language differences. In 1953, Wynn-Lai wrote a Book Linguistic Contact from the perspective of structural linguistics $\mathrm{He}$ first proposed the conceptual framework of contrastive analysis. On the basis of the theory of structural linguistics, Rado published the world's first monograph on contrastive linguistics Intercultural Linguistics in 1957, which marked the further development of contrastive linguistics. With the emergence of textual linguistics, Hartmann and Connor wrote literature on textual contrastive studies from the perspective of textual linguistics, discussing the relationship between language contrast and translation. At the same time, some scholars used generative semantics and systemic functional grammar to make a contrastive study of bilingual articles.

\section{B. Domestic Research}

The contrastive studies between Chinese and English in China is acknowledged to have gone through four distinctive major stages of development by many esteemed contrastivists. The first stage is the starting stage that begins from 1898-1940s MA' s Grammar is the first textbook of Chinese grammar written by a Chinese scholar, Ma Jianzhong, who published it in 1898 , and is generally attributed as the "germination of modern linguistics in China." His book symbolized a new stage of the Chinese grammatical studies, which broke through the traditional studies and shadowed all the previous achievement which focus on the explanation of ancient contexts. Therefore, 
the book transformed the academic status of Chinese grammar from accessory state into a domain state. In 1924, Li Jinxi published A New Grammar of Mandarin Chinese took the modern Chinese as the object of study. A New Grammar of Mandarin Chinese is a valuable contribution to the modern history of the Chinese grammar research with its new grammatical conceptualization: the sentence level grammatical conception, the definition and classification of six sentence elements and seven word cases of nouns and pronouns, the diagrammatic sentence analysis method, the word-class classification. Lin Yutang, Chinese writer, translator and linguist, in his book Kaiming English Grammar published in 1933, defined the grammar as "the science of expression" and declared his use of the contrastive method between English and Chinese. "In a way, the Kaiming English Grammar contains within its covers the administration of a new comparative grammar of the English of the English and the Chinese language. This reduces itself to a comparison of English and Chinese notional categories and the different means employed to express these notions. ...And where common notions exist in both languages, as in the majority of grammatical categories, the most interesting thing would be to show how differently the two languages express these notions. English grammar, presented in this way, therefore, teaches the English ways of thinking and expression (Lin Yutang, 1933). "With his wide erudition and linguistic talent, Lin Yutang made a great contribution to explaining usages, contrasting the ways of expressions and translating examples.

The second stage is that the development of English-Chinese Contrastive Studies are stranded, which is from 1950s to 1960s. In this stage, the Chinese contrastive achievements accumulated in the first half of the century were soon depleted and a good opportunity of pushing contrastive advantage and making major theoretical breakthroughs like those in the West was let slip by as a consequence of many social political movements or events that hurt, convulsed or even crippled the country economically and socially. However, there are still some achievements are deserved to be documented, such as: Wang Li' s " On the Relationship Between Native Language Learning and Foreign Language Learning" in 1954, "Provisional Schema" in 1956 and Lü Shuxiang' s English Learning for Chinese Speakers reprinted in 1962.

The third stage is revival stage that is from 1970s to 1980s. This stage is represented by Lü Shuxiang' s speech entitled "Study Grammar by Way of Contrasts" at the Beijing Institute of Languages in 1977, which epitomizes the age of the contrastive linguistics as an Independent academic discipline pursued by scholars with definite theoretical and practical purposes. The method of contrastive study was reiterated by Lü Shuxiang in his other publications. "The character of a thing cannot protrude until compared with others. For instance, the human traits such as walking upright on his feet, tool making and language speaking are perceived when compared with other animals. The same is true of languages. The features of the Chinese language are thrown into the light in comparison with non-Chinese languages, the features of the modern Chinese stuck out in contrast with the classical Chinese. And the features of mandarin Chinese leap to the eye when compared with the regional dialects of Chinese. The studies on phonetics, lexicology and grammar can be all undertaken from a comparative approach. " [1]

Since 1990s, the contrastive studies between Chinese and English in China has been into a vigorous stage. In this stage,there are four important events deserved to be documented in the history of contrastive research in China. First, A Collection of Papers in English-Chinese Contrastive Studies edited by Yang Zijian and Li Ruihua was published in 1990 and in the foreword to it. Lü Shuxiang pointed in the direction of new possibilities and potentials for contrastive research in China. Second, the first mature theoretical framework of contrastive studies was initiated in CE-EC Contrastive Studies and Translation by Liu Miqing, former professor of the Chinese University of Hong Kong, in 1991. Third, An Introduction to Contrastive Linguistics by $\mathrm{Xu}$ Yulong in 1992 marked that contrastive linguistics had gained a foothold as an independent discipline in China. Fourth, after four years preparation, the China Association for Comparative studies Chinese(CACSEC) was authorized by the government department and established in 1994 (Xu Yulong,2010). This stage witnesses the mushrooming of contrastive linguistic studies in China featured by the broadening of research field, diversification of research topics and research methods, and proliferation of publications, endorsed research projects at collegiate, provincial, ministerial or national levels and sponsored symposiums or workshops on contrastive linguistics After the 1990s, contrastivists are more willing to beyond the limits of conventional ways and attempt to study English-Chinese linguistics reality from novel perspectives.

\section{Deficiencies and Prospects}

With the introduction of various foreign theories and methods, the contrastive study of English and Chinese in China has seen a diversification of theories and methods. While actively referring to foreign linguistic theories and methods, the researchers also closely connected with the Chinese practice, and tried to explore the theoretical framework and common basis of comparison between English and Chinese. The research level was greatly improved, and remarkable achievements were made in revealing and explaining the similarities and differences between Chinese and English at different levels. In the meanwhile, at present, although the study of English-Chinese language contrast and translation in China has developed rapidly and achieved abundant results and many productions are obtained from macro and micro perspectives, the macro-research is limited in the following several aspects: 1) The methodology and history of the discipline are not enough, and the discipline is not mature enough; 2) The phenomenon of imitation in English-Chinese contrastive studies and translation is still widespread and does not really integrate with the reality of China. There is no discipline of contrastive and translation between English and Chinese with Chinese characteristics; 3) In Big Data Era, corpus-based research methods of English-Chinese contrastive studies and translation are inadequate; 4) Lack of cultural comparison between English and Chinese in textual level. These are the challenges facing scholars. It still has a long way to go to promote researches and development of English-Chinese contrastive studies and translation. 


\section{ReCEPTION AESTHETICS AND AESTHETIC Linguistics}

Reception aesthetics is a new methodology of literary research proposed by H.R. Jauss in the 1960s, which emphasizes that the educational and entertainment functions of works should be realized by readers. Reception aesthetics focus on the readers' acceptance of works, reaction process and readers' aesthetic experience of reading, and the acceptable effects in social functions of literature. Reception aesthetics put the aesthetic experience in history and the social conditions through the study of the creation and acceptance of dynamic interaction process between authors, works and readers. The "expectation horizon" inherited the traditions of Heidegger and Gadamer's modern hermeneutics. Jauss believed that the perspective of expectation not only determined the standard of readers' judgment on the content and form of works, but also involves the basic attitude and overall evaluation of the works. Receiving is the process of creating works by readers' aesthetic experience, which excavates various connotations in works. The art value of works are not permanent, but have a history value that is constantly accepted by readers in different societies and historical periods. Beauty exists only when it is accepted. So do classics. Readers' accepting activities are restricted by their own historical conditions and the scope of works, so they cannot follow their inclinations. The author establishes a dialogue relationship with readers through his works. When a work appears, there is a level of anticipation, the expectation of what to read from it. The reader's expectations establish a frame of reference on which the reader's experience interacts with the author. The level of expectation is determined not only by the genre of literature, but also by the experience of readers who have read such works before. The value of the work lies in its inconsistency with the readers' expectation level and its aesthetic distance.The initial research target of reception aesthetics is literary works, which studies readers' acceptance of literary works in the process of reading. Jauss gradually established a reader-centered view in his later studies, which is also one of the main views of reception aesthetics. After absorbing the thoughts of Romain, aesthetics of reception confirms the role of readers in the reconstruction of literary works, believing that the value of a literary work is not endowed by the author, but completed by the reader. Secondly, reception aesthetics also puts forward a dynamic perspective of expectation, believing that readers' "pre-understanding" of literary works is not invariable, and may be influenced by readers' knowledge background and time background. Different readers at different times have different perspectives of expectation. Finally, Ethel (1987:97) believes that the meaning of a work can only come into being in the process of reading and the meaning is the product of the interaction between the work and the reader. Ethel believes that the text has a "calling structure", which enables the reader to supplement the blank space in literary works, so as to truly realize the value of literary works.

Aesthetic choice of language refers to the choice approaching the appropriateness infinitely in a group of language elements with slight differences, made by the language subject to achieve the best effects in practical and aesthetic purposes from the intention of aesthetics. The aesthetic choice of language includes the aesthetic choice of signals, channels, language variants, styles, types of communication, discourses etc.

\section{COMPARISON AND TRANSLATION STRATEGIES}

\section{A. Comparison Between English and Chinese in Character Reporting Based on Reception Aesthetics and Aesthetic Linguistics}

Chinese characters reporting in foreign media have their own fixed and frequently used words. (1)title of the character: "the hottest artist on the classical music planet" "a cultural ambassador" "cultural tourism ambassador" "a pioneer of a great cause" "Five Great Asian Divas" "Pioneering Hong Kong movie producer" "Chinese martial arts novel grandmaster"" the JRR Tolkien of Chinese literature and the grandfather of martial arts novels" (2) adjectives equipped with the same meaning frequently used in text: seductive,addicting,spellbinding, enamored, intriguing, fascinating, enthralling, riveting, captivating, (3) others' evaluation: acclaim, loud, etc.

(4)Show someone is influential: 1) Lang Lang is a classical pianist who has been making waves. 2) Tributes to the beloved penman poured in from far and wide. 3) Accolades have long poured in for his contribution to literary arts not only from China but also from the British and French governments -there is even an asteroid named after him. 4) Tributes have been pouring in, remembering him for a lifetime of designing iconic structures worldwide. These frequently used expressions demonstrates fixed model of the expressions of character reports.

Voice is the physical shell of language. According to the theory of aesthetics and aesthetic linguistics, it is found that both English and Chinese phonetics have aesthetic attributes, presenting the beauty of sound, rhyme, cadence, rhythm and continuity, etc. The main differences are embodied in the way of presenting phonetic beauty and the use frequency of aesthetic elements. The aesthetic attribute of phonetics reflects the aesthetic characteristics of language and contains different aesthetic choices of Chinese and English. The beauty of pronunciation is determined by the social attributes of pronunciation and is the result of the long-term development of language. In a character reporting of Louis Cha, his words are known for their poetic quality, like this sentence from The Return of the Condor Heroes. "Look at those pale clouds, gathering together, drifting apart, gathering and drifting human unions and partings, are just the same." The bold part generate a kind of harmonious formal beauty. Through the repetition of phonemes, the fluctuation of stress, the echo of intonation and the continuity of syllables, it forms the basic elements of producing phonetic beauty. These elements are organically coordinated to give the target reader psychological satisfaction in the dialectical unity and jointly contribute to the beauty of phonetics.

Translation is not only to express the meaning of one language into another language, but also to express the thoughts of one culture into another culture. Therefore, translation is both linguistic and cultural. When deal with this sentence---Lang's physical poses - which never really bothered me but drove others up the wall - are now less exaggerated, 
and they serve as a reflection of where the music is going, not a distraction. The bold part may confused us with "A cornered dog will leap over a wall", unless we know this American idiom. It has been used for over fifty years. When introducing Chinese people, the foreign media respects the reading expectation and acceptance preference of target readers, make a reasonable choice to achieve publicity effect. So they use some language items to produce an impression of an original language. The using frequency can not only verify the standard degree of the translation, but also reflect the quality of the text. E.g. "Run Run was like a Louie B. Mayer type - he had a very paternal attitude toward his stars". The foreign media make an amplification just because the foreign readers are more familiar with Louie B. Mayer. In addition, in the language system, culture loaded words can best reflect national distinct cultural personalities. "He liked to be photographed in a tai chi exercise pose, wearing the black gown of a traditional mandarin." Tai Chi, as Chinese system of slow meditative physical exercise designed for relaxation and balance and health, preserves the cultural characteristics of the original text and conforms to the reading habits of English readers. So do the title of films and the characters, for example, "Directed by Hong Kong auteur Wong Kar Wai, "The Grandmaster" is loosely based on a true story about Ip Man, the martial artist who taught a young Bruce Lee the fundamentals of Wing Chun kung fu." and "The Book and the Sword" ,"Sword of the Yue Maiden" etc.

A good news report can bring people a kind of aesthetic enjoyment. Create a corresponding image can help achieve this effect. In fact, the audience's reading and evaluation of news works is an aesthetic consumption, which includes not only external attention, but also their own aesthetic consciousness. An effective and revelatory approach is metaphor. Compared domestic reports with foreign reports, there are big differences in shaping the language.(1)"Rhapsody In Blue" with Herbie Hancock is the centerpiece of the album.(2) The seeds of decline may have been geopolitical as well as micro-economic.(3) "Architecture is stones and brick, concrete and steel. Architecture has to endure."(4) Although Shaw was a renowned talent spotter, he missed the potential of Bruce Lee, whose TV career started in the late 1960s.(5) "He should rightly also be given credit for pioneering a form of Asian musical film and for putting Hong Kong on the global cinema map."(6) "Architecture must have integrity, like a friend."Furthermore, figuratively from different perspectives, the following report examples in the same text describethe distinct music characteristics of the trio and its intuitive feeling, which is more three-dimensional than the tiling description in Chinese reports.(1)The third movement was not what I would have expected from this virtuoso; it was moderately slow and relaxed with limpid dynamic control, a flowing river rather than the usual stoked locomotive.(2)Lang made it an experience of contrasts as varied as the weather itself - turning on the hammering drive in "August" and "September," ruminating through "April" and drifting through "May" with lots of expression.(3) the wild, impressive extroverted flourishes, the melting touch that he applied to the Trio sections, a gorgeous rippling waterfall effect in the third Scherzo, the bombs-away codas.
The news is not a complete copy of the information, but a selective report. Although it is the true records, we can't do it without skilled language. When we receive information, what we know and feel comes not from our inner consciousness, but from external influences. News has its own frame in editing. When we enjoy the satisfaction and pleasure brought by news, the subject is immersed in the world of "other", and we gradually take the vision of "other" as the standard of our aesthetic judgment. The two perspectives that are often used in setting off characters are from other famous character's evaluation and from the characters' works. The former often applies to domestic reports (eg.1.2.3), while the latter often appears in foreign reports (eg.4).(1)Jack Ma, the owner of Chinese e-commerce giant Alibaba and a friend of Jin, described his passing as "a huge loss for Chinese people around the world.”(2)Hong Kong's top official, Chief Executive Carrie Lam, called Cha "a learned man and an acclaimed writer" in a statement, adding that his works "inherited the tradition of Chinese classics with the integration of history and culture."(3)Though Bruce Lee died 40 years ago, the story of his grandmaster, Ip Man, continues to shape the kung fu legend that endures today."I think we're trying show to all the audience in the world to revisit this lost heritage and what is the true spirit of kung fu," Leung said. Bruce Lee and kung fu's influence can be seen all over today's pop culture. Lee was the catalyst for the collision of Asian and American pop culture, inspiring generations to learn kung fu.(4)Hollywood films have for years been doing strong business in China. But rarely does an American film perform better in China that it does stateside. That's been the case for "Life of Pi," filmmaker Ang Lee's adaptation of the bestselling 2001 novel about a young boy trapped in the middle of the ocean on a raft with wild animals. The fame of the film reflects the author's talent. He is a master at epic filmmaking, as beautifully illustrated in Crouching Tiger, Hidden Dragon. $\mathrm{He}$ is brilliant at evoking emotional resonance, as he demonstrated in Brokeback Mountain. That means, a masterpiece is produced by a master filmmaker. Similarly, "There are almost no fight scenes in Hollywood movies today that don't rely on Asian martial arts." And that's directly attributable to these martial arts movies that the Shaw Bros. brought over in the 1970s.

In foreign reports text, several methods are used to strengthen the artistic effect: (1) Use of antonyms: "At a time when so many people in the world of design are concerned about being timely, his work will always be considered timeless."; Mr. Chow's biggest success, and Mr. Shaw's most notable loss, was his decision to bankroll Bruce Lee; "he has given this century some of its most beautiful interior spaces and exterior forms". (2) Use of emphasis sentences: I think not every bone but I can say every inch of my body I got cut burned twisted. (3)Use of double negation: Compared the sentence "It shows that you must never fear to be audacious because in the end, when you bet on intelligence and beauty, it's a success" with "It shows that you must to be audacious...", though they have the same meaning, the tones are quite different. (4) Use of verbs: Ever excited about all he has coming up, and so honored by his new title, the pianist sat down for an interview about his new album, his new initiative, and what else has coming up; While Shaw didn't create the 
kung fu movie, he was quick to capitalize on the genre's trendiness and used a modernized studio system and centralized production techniques to pump out films quickly, beating out rivals to satisfy audience demands. Apart from this, details are meticulously depicted so as to enhance a picture sense or create a visual sense. (1)The stunt involved jumping between two walls of a castle and then swinging through the branches of the trees below. On the second take, the action star crashed straight down and landed on a rock. (2)Last month he was at the Grammy Awards in Staples Center pounding out splatters of notes within an overblown production of Pharrell Williams' catchy hit song, "Happy."

The western reportsoften use objective descriptive language, while China lays particular stress on passionate narrative language.Western mediapour out purposeful words flowing directly from entertainment news events. They believe that good news should be descriptive and reproduce the scene, sound and atmosphere of the event; Good news should follow the same pace and tone as the story itself. Domestic news reports often use emotional and subjective language. Adjectives are often used, and of course, it shows the strong lyricism.Known in Chinese as Deng Lijun, Miss Teng's surname was the same as that of Deng Xiaoping, China's Paramount leader. So she was jokingly known as Little Deng, And in some circles she was nearly as famous as he. Even peasants in the countryside were familiar with her songs and brightly colored costumes. Many other foreign singers became popular in China, but none captured the hearts of the country as she did. From Singapore to Japan, Miss Teng was a superstar and brilliant linguist. She sang in Mandarin Chinese, Cantonese Chinese, Japanese and English, and in recent years she had lived in Japan and became popular among both the young and the old. "peasants" "from Singapore to Japan", depicts the image of a "superstar". "Brilliant linguist" is synonymous with "polyglot" and "multilingualism", and sound similar to "sang in Mandarin Chinese, Cantonese Chinese and English". "Wherever there are people, there will be Teng's songs." A popular saying was that Old Deng ruled the day and Little Teng the night. Her transnational success was attributed to her multilingualism (she sang in Mandarin, Cantonese, Japanese, and English), her clear and sweet vocals, and her heart-rending love songs, which appealed to both young and old in such far-flung locations as China, Malaysia, Singapore, Indonesia, and Japan. -- "A museum to commemorate her was established in Taiwan, and she also inspired a theme restaurant in the Chinese capital, Beijing. All these shows that people keep Teng's memory alive in a multitude of ways, and she will always be one of the shining stars in Chinese culture.

Chinese reports are good at macro reporting, while western news reports are usually from micro perspective. The essence of macro reporting is to proceed from the overall situation and not to transmit specific information of the event. Micro perspective is essentially a description of details, capturing the emotional and linguistic changes of the reporting objects. Chinese and western character news reports have their own unique features. The portrayal of The Rock and Roll Hall of Fame reflects the character's fame indirectly. Profile description can stimulate people's imagination and is a useful complement to positive description of characters. Apart from this, profile description is conducive to the expression of character. The Rock and Roll Hall of Fame is a western achievement award dedicated to some of the most recognizable and influential artists, producers and, in important ways, those who have influenced the music industry as a whole. It takes into account the contributions of selected musicians to the history of rock and roll, and is a testament to their achievements. One of the most demanding conditions is that the nomination must be made more than 25 years after the release of the first album. I.M. Pei (PAY), one of the 20th Century's most prolific architects, a versatile, globe-trotting architect has designed municipal buildings, hotels, schools and other structures across North America, Asia and Europe and revived the Louvre with a giant glass pyramid and captured the spirit of rebellion at the multi-shaped Rock and Roll Hall of Fame. His buildings added elegance to landscapes worldwide with their powerful geometric shapes and grand spaces. Among them are the striking steel and glass Bank of China skyscraper in Hong Kong and the Fragrant Hill Hotel near Beijing. Hard-working, energetic, genuinely humble, exceedingly charming---"These are not always the attributes of great artists, but they were often used by multiple colleagues, rivals and critics, who hailed Pei in his lifetime as a world-class architect - and a most endearing man. ". The use of adjectives describes the character and quality of the character. In an interview, he stressed, " I also think architecture can reach a level where it influences people to want to do something more with their lives. That is the challenge that I find most interesting." Pei's unspoken attitude towards clients was, "If you want to do a piece of junk, I'm not your architect." But Pei was far too diplomatic and discreet to say such a thing out loud. Pei's working methods included careful research and immersion, steeping himself in the surrounding environment. This kind of expressive discourse expresses the speaker's subjective wishes and feelings, and expresses their feelings towards reality, personal feelings and attitudes towards life. The language has an aesthetic function, and translating such texts is a process of re-creation in another language, inevitably carrying with the translator's personal behavior and performance style. And in the translation process, we must considerthat although his background was in engineering, but his architecture reflects a beautiful sense of engineering

\section{B. Translation Strategies Between English and Chinese in Character Reporting Based on Reception Aesthetics and Aesthetic Linguistics}

The audience's aesthetic expectation should be considered when it comes to report character news. Explore their yearning and pursuit for cultural tastes so as to lead the correct social value orientation. From the perspective of reception aesthetics, what really touches the audience is the continuous innovation and resonating local connotation. The real products with humanistic charm must take readers into consideration.

The film release is an ancillary effect of China's space rivalry with other countries and the backdrop is one of the basic operations of foreign media reporting on China -- trying to find social or political motivations in many entertainment or commercial events, so as to see the bigger picture and reflect current trends from the events. This kind of writing and reporting is very enlightening, but its operation seems to be 
unconsciously turning into a kind of political correctness in foreign media coverage on China. Inevitably, sometimes it is the case that write a new word without sorrow and barely say sorrow and let readers see some very far-fetched connections. So in the process of winning the world's attention and applause, Chinese films rely on their own high artistic level and the brilliance of human nature, rather than being subconsciously regarded as the appendage of something else. This is a great inspiration for reporting Chinese films and characters. The fame of a character is usually set off by the sensation of a film, and the popular of a film equally means that the character has great influence and there are great similarities and integration between the two reports. Jackie chan's bold attempt to play a nameless grandpa full of childlike fun overturned his inherent image in the audience's mind for the first time, successfully completed the role conversion, and brought the audience a new aesthetic experience. Properly complete the local adaptation. It takes the readers into consideration. In the film creation process, some familiar plot and story elements are consciously adapted to arouse readers' response and thinking. From the perspective of scene setting, the film interprets such a fantasy and warm "Chinese story" to resolve cultural differences through film adaptation. To transform "potential readers" to "actual readers", film creation and character reports should fully consider the subjective and objective factors of the audience, grasp the audience's psychological activities and stimulate the interests of the audience in watching movies.

From the perspective of reception aesthetics, the translators should consider the aesthetic temperament, understand the differences between Chinese and English in cultural background and language expression, and flexibly use of foreignization and domestication translation strategy according to the specific content so that the target text embodiesthe aesthetic characteristics.Due to the differences between Chinese and western culture and way of thinking, there are lots of differences in language aesthetic choice between foreign media and domestic media when reporting characters. We must pay attention to them so that they can meet the aesthetic needs of specific groups and be more acceptable to the public.

With comparisonbetween English and Chinese in character reporting based on reception aesthetics and aesthetic linguistics made, the author summarizes the following several translation strategies:

(1)In the process of Chinese-English characters reporting, appropriate rhetorical devices should be used. Metaphors and appropriate and fixed images should be added to make the characterization more three-dimensional and vivid, and vice versa---delete metaphors or fixed images.

(2) In the process of reporting film characters in foreign media, we usually make use of other people's evaluation, specific examples and the foil of works to make the characterization more realistic. When reporting characters in Chinese, we tend to deal with them from the perspective of the first protagonist.

(3) In the process of reporting the death of a person, pay emphasis on his life stories and insert images or pictures of living people in the text. On the contrary, in the domestic media, more attention is paid to the rendering of feelings of mourning in the text.

\section{CONCLUSION}

Under the guidance of the theory of reception aesthetics and esthetic linguistics, translators should take into account the readers' expectation horizon, fill in the "blank" appropriately, and translate into the language accepted by the target language readers. Considering the unique language expression habits, cultural background knowledge and thinking mode of target readers, the translation process is in line with the understanding and acceptance ability of target language readers to the greatest extent.

\section{REFERENCES}

[1] Wang Wenbin. Contrastive Linguistics: the Essentials of Language Research [J]. Foreign Language and Foreign Language Teaching, 2017 (05).

[2] Hans Robert Jauss. Reception Aesthetics and Reception Theory [M] Shenyang: Liaoning People's Press, 1987.

[3] Pan Wenguo. A hundred years of comparative studies between Chinese and English [J]. World Chinese Teaching, 2002(1).

[4] Pan Wenguo, Tan Huimin comparative linguistics: historical and philosophical reflections $[\mathrm{M}]$. Shanghai: Shanghai Education Press, 2006.

[5] Zhu Liyuan. Introduction to reception aesthetics [M]. Hefei: Anhui Education Press, 2004.

[6] Si Xianzhu. Comparative study of Chinese and western views on translation $[\mathrm{J}]$. Foreign Language and Foreign Language Teaching, 2005 (3).

[7] Qu Yan. Linguistic and cultural dimensions of English to Chinese translation [J]. Journal of Foreign Languages, 2006(5).

[8] Liu Miqing. Contemporary Studies of Translation.[M].Bei Jing: China Translation \& Publishing Corporation, 2005,11.

[9] Wang Lixin. A diachronic comparative study of Chinese and Western translation methodologies $[\mathrm{J}]$. Journal of Harbin University of Technology (Social Science Edition), 2002 (02): 103-106.

[10] Ma Jianzhong. Ma Shi Wen Tong--Chinese Grammar Series[M]. Beijing: The Commercial Press, 1983.

[11] Pan Wenguo, Tan Huimin. Contributions of Wilhelm von Humboldt and Benjamin Lee Whorf to the Founding of Contrastive Liguistics.[J].Journal of East China normal University (philosophy and Social Science Edition),2005(06):75-80

[12] Hu Shuzhong. A comparative study of English and Chinese rhetoric [M] Shanghai: Shanghai Foreign Language Education Press, 1993.

[13] Luo Xuanmin. A comparative study of discourse analysis between English and Chinese [M]. Changsha: Hunan People's Publishing House, 2001 